Introducción

\title{
"Dulce Padre Pio, estoy aquí en oración... para rogarte que vengas en nuestra ayuda y nos protejas del covid"1. Algunas reflexiones teóricas sobre la relación entre religión y COVID 19
}

\author{
Francesco Gervasi², SIMONA SCOTtI ${ }^{3}$
}

\section{La religión como estrategia para gestionar los momentos críticos de la existencia}

Ya desde hace algunos años, varios estudiosos han destacado que las sociedades industriales han sido sustituidas por aquellas "del riesgo global" (Beck, 1998), es decir sociedades caracterizadas por la producción y difusión de riesgos, principalmente de tipo manufacturado ${ }^{4}$, que no están "restringidos espacial, temporal o socialmente" (Giddens, 2014, p. 124). En esta situación, entonces, como sostiene Giddens (2014, p. 124), la "gestión del riesgo es el rasgo principal del orden global", en el sentido que esta tarea se convierte en una de las más importantes, entre las que gobiernos y ciudadanos deben (o, mejor dicho, deberían) de realizar dentro de las sociedades globalizadas contemporáneas.

Tomando como punto de partida las tendencias arriba mencionadas, el presente número de la revista Comparative Cultural Studies - European and Latin American Perspectives, titulado "Religiones y riesgos globales. Resistencias y reconfiguraciones del factor religioso en la época de la COVID 19", se enfoca en el tema de la relación entre religión y COVID 19. Más específicamente, la pregunta general a partir de la cual se desprenden las reflexiones que presentaremos a continuación es: ¿Cuáles han sido las respuestas adoptadas por las religiones institucionales, las religiosidades individuales y las populares, para enfrentarse a los problemas generados por la pandemia de la COVID 19?

Sin embargo, antes de presentar los textos de los estudiosos que han participado en este número monográfico, vale la pena contestar a otra pregunta, es decir: ¿Por qué, en este número de Comparative Cultural Studies - European and Latin American Perspectives, hemos decidido enfocar la atención precisamente en la relación entre religión y COVID 19? El mensaje subyacente de esta pregunta puede ser aclarado a través de esta otra inte-

\footnotetext{
${ }^{1}$ Encontrado en: https://www.padrepiodapietrelcina.com/preghiera-protezione-guarigione-coronavirus/. Consultado el 12-02-2021. En el sitio, la oración aparece en italiano: "Dolcissimo Padre Pio, sono qui in preghiera...per implorarti di venire in nostro aiuto e proteggerci dal covid".

${ }^{2}$ Profesor-investigador de tiempo completo, Facultad de ciencias de la comunicación, Universidad Autónoma de Coahuila. Email: francescogervasi@uadec.edu.mx

${ }^{3}$ Jefa de redacción de la revista Religioni e Società y experta de Sociología de las religiones en la Universidad de Florencia. Email: simonascotti@inwind.it

${ }^{4}$ Es decir, aquel tipo de riesgos que son consecuencia del desarrollo social, científico y tecnológico humano. Lo anterior, obviamente, no excluye la persistencia, en nuestras sociedades, de muchos riesgos de tipo natural. Establecer si la COVID 19 pertenezca al primer tipo de riesgos o al segundo es una tarea que no forma parte de los objetivos de este trabajo.
}

Copyright @ 2021 The Author(s). Open Access. This in an open access article published by Firenze University Press (www.fupress.net/index.php/ccselap) and distributed under the terms of the Creative Commons Attribution 4.0 International License. The Creative Commons Public Domain Dedication waiver applies to the data made available in this article, unless otherwise stated. 
rrogante: ¿cuál es el papel que tiene el factor religioso en los mecanismos de defensa que los seres humanos utilizan para enfrentarse a situaciones de crisis como, por ejemplo, una pandemia mundial? ¿Es un papel importante o, al contrario, inhútil y hasta negativo?

Para contestar a estas preguntas, conviene tomar como punto de partida la función de interpretación y de legitimación del mundo que, típicamente, la religión ofrece a los seres humanos. Peter Berger (1977), por ejemplo, definía la religión como dosel sagrado, justamente para referirse a esta capacidad que tiene de legitimar la realidad social, entre otras cosas, integrando (atribuyéndoles un significado y, consecuentemente, legitimándolos) los momentos críticos de la existencia en un cosmos dotado de sentido. Parecida a la anterior es la idea de Parsons (1981), quien sostenía que la religión, en las sociedades contemporáneas, tiene el papel de mantener en equilibrio el sistema social, sobre todo en aquellas ocasiones en las cuales, eventos dramáticos como la muerte, la enfermedad y las injusticias en general, ponen en riesgo este equilibrio. Según Parsons la religión ofrece una explicación de estos eventos, volviéndolos, por lo tanto, comprensibles y aceptables, sobre todo gracias a la idea de un orden sobrenatural que tiende a compensar, en otra vida, los momentos negativos experimentados en la existencia en la tierra. En palabras del sociólogo estadounidense: "la concepción de un orden sobrenatural se utiliza para delinear un reequilibrio "compensatorio", es decir, un Ausgleich en una esfera trascendente, que en el caso más común es la vida después de la muerte. Entonces es posible pensar que una buena fortuna inmerecida y un sufrimiento inmerecido se compensarán en otro lugar" (Parsons, 1981, p. 379).

Sin embargo, vale la pena destacar que la religión no sólo ofrece una posible interpretación (y consecuente aceptación) de los momentos negativos de la existencia, sino que también representa una herramienta muy poderosa que los seres humanos utilizan para enfrentarse a la amenaza de que estos eventos ocurran. Por ejemplo, Ernesto De Martino (2003), concentrando su atención en las prácticas de la religiosidad popular en el extremo sur de Italia, sostenía que los rituales mágico-religiosos eran utilizados sobre todo por parte de las personas más vulnerables, para superar los momentos críticos de sus existencias, actualizando (a través de los rituales) aquellas prácticas que, en la meta-historia (es decir en una época mítica), permitieron superar el mismo momento crítico al cual, en la situación actual, ellos tenían que enfrentarse. Según De Martino (2003), entonces, la religión, entendida justamente como conjunto de herramientas mágico-rituales, tiene un papel fundamental en la reducción de la incertidumbre y del caos, en cuanto es capaz de establecer "un régimen de existencia protegido, que, por un lado, nos defiende en contra de las irrupciones caóticas del inconsciente y, por otro lado, oculta el devenir permitiéndonos de permanecer en la historia como si no estuviéramos allí” (p. 97).

Otra pregunta a la cual conviene contestar en esta introducción es: ¿cuál es el papel de la religión en un mundo, como el actual, dominado cada vez más por la racionalidad, por el conocimiento científico y por la tecnología, que, desde los tiempos de la ilustración, prometían alejarnos "del miedo y [que] nos aproximarían a un mundo libre de la ciega e impermeable fatalidad" (Bauman 2007, p.10)? La respuesta a esta pregunta debe de tener en cuenta que estas previsiones han fracasado y que "en el escenario de la modernidad líquida, la lucha contra los temores ha acabado convirtiéndose en una tarea para toda la vida" (Bauman, 2007, p. 10), como lo demuestran, entre otros ejemplos, la amenaza continua de una guerra nuclear, las hambrunas, los atentados terroristas, las epidemias y pandemias, las nuevas y viejas enfermedades, la contaminación, etc. 
En este contexto, entonces, la religión entra en juego en cuanto estrategia, individual o colectiva, utilizada para enfrentarse a todos estos peligros que la ciencia no ha logrado eliminar de nuestras vidas. Como destacado por Voyé (1999, pp. 278-279):

Al contrario de lo que se esperaba durante la modernidad, hoy nos enfrentamos a la incapacidad de la ciencia para resolver todos los problemas (pensemos en la pobreza, la guerra o la enfermedad y en la muerte, por ejemplo). (...) la ciencia, muy a menudo, no tiene una importancia directa para los individuos ya que sus desarrollos no tienen una aplicabilidad inmediata, porque no pueden ofrecer las respuestas urgentes que la gente necesita para enfrentarse a sus problemas concretos y particulares aquí y ahora. (...) Lo anterior estimula a la gente a buscar una solución diferente (o al menos una esperanza de solución) con respecto a lo que ofrece la ciencia. En este sentido, la religión popular representa un importante recurso. La gente realiza sus peregrinajes, por ejemplo, con la esperanza de solucionar o evitar problemas de salud personales o de sus familiares, para reconstruir la armonía familiar, para encontrar trabajo o para pasar un examen.

Después de haber destacado la actualidad de la religión, en cuanto recurso estratégico utilizado para esperar solucionar (o para esperar evitar o aceptar) algunos de los peligros tradicionales y modernos que siguen afectando a la humanidad, a continuación conviene recordar brevemente algunos de los principales problemas que, hoy en día, la COVID 19 ha implicado para el ejercicio colectivo (principalmente) de las prácticas religiosas.

\section{Problemas, resistencias y reconfiguraciones del factor religioso en la época de la COVID 19}

La COVID 19, ya desde casi un año, ha implicado cambios muy importantes en nuestras vidas. Seguramente, uno de los principales tiene que ver con el distanciamiento social, el cual ha provocado una sensible disminución de las interacciones y de los contactos que las personas pueden tener con los demás. Esta importante disminución de las interacciones, obviamente, ha tenido un impacto también en las formas de expresión de la religiosidad, tanto aquellas institucionales como las de tipo popular. A pesar de que ya desde hace muchos años, varios autores han destacado una tendencia hacia la privatización de la religión, para muchos creyentes sigue siendo fundamental poder vivir su experiencia religiosa con otras personas. Como destacado por Hervieu-Léger (2003, p. 143), "Para estabilizar los significados que producen con el objetivo de otorgar un sentido a su existencia cotidiana, los individuos raramente pueden conformarse con sus propias convicciones. Ellos necesitan encontrar en el exterior la garantía de la pertinencia de sus creencias". Además, el mismo significado etimológico del término "religión", que es justamente "ligar o amarrar", nos remite a la idea de que su principal objetivo es el de favorecer la relación y la unión entre personas.

La pandemia ha provocado una disminución (o interrupción hasta nuevo aviso) de muchas prácticas religiosas colectivas, como las misas, las fiestas populares dedicadas a los santos patronos, la celebración de matrimonios y funerales, la simple convivencia entre miembros de un mismo grupo religioso, las actividades sociales de muchos movimientos, grupos e iglesias, etc. A la luz de este escenario, las instituciones religiosas y los creyentes, en muchos casos, han tenido que reorganizar estas actividades de manera virtual, con todos los problemas que lo anterior implica. El ejemplo más evidente de estos problemas tiene que ver, seguramente, con las dificultades que encuentran los creyentes que no tie- 
nen acceso, o no saben manejar, las herramientas tecnológicas utilizadas para realizar estas prácticas de manera virtual. Y el anterior no es el único, porque existe también un problema más sustancial que tiene que ver con que, en las prácticas virtuales, para algunos/as desaparece una de las características principales de la religiosidad practicada en espacios físicos, es decir la posibilidad de vivir esta experiencia a través de los sentidos, los cuales, como destacado en este ejemplo relativo a la religiosidad popular por Berzano (2014, pp. 78-79), se expresan mediante:

el perfume de los inciensos y de la cera de las velas; las manos que tocan los mármoles, los balaustres y los relicarios; los cantos, los sonidos del órgano y el fondo de las oraciones cuchicheadas de los peregrinos; el peregrino de pie o arrodillado, las manos levantadas o juntas, la cabeza apoyada en el gran relicario que guarda el cuerpo del santo.

A continuación, mencionaremos tres ejemplos encontrados en el contexto mexicano que reflejan, de manera bastante evidente, las reconfiguraciones de las prácticas religiosas que se están dando en todo el mundo, como consecuencia de la difusión de la COVID19.

El primer ejemplo nos lo ofrece la famosa representación del Viacrucis de Semana Santa en Iztapalapa que, el año pasado, después de muchas resistencias por parte de los organizadores, se ha tenido que realizar, por primera vez en su historia, a puertas cerradas. Otro ejemplo más reciente tiene que ver con la nueva manera de vivir el Miércoles de Cenizas, unos de los rituales más importantes dentro del catolicismo. A este propósito, el diario Excelsior (Velasco, 2021, 17 de febrero), el 17 de febrero, ha publicado una nota con el siguiente titular: "Con ceniza en bolsa y misas grabadas, así vive Edomex el Miércoles de Ceniza”, explicando que, en las pocas iglesias abiertas, los sacerdotes estaban colocando la ceniza en la cabeza de los pocos feligreses que llegaron ese día, en lugar que en la frente, y que, después, les entregaban unas bolsitas con adentro más ceniza, para que la distribuyeran entres sus familiares. Por supuesto que los cambios y las dinámicas de adaptación a la pandemia han involucrado a todas las religiones y no sólo al catolicismo, como lo confirma, entre otros, el siguiente titular del Vanguardia de Veracruz (2020, 18 de diciembre): "Pastores reciben reconocimientos tras capacitación por COVID-19", mencionando que, en Papantla, en el Estado de Veracruz, se entregaron reconocimientos a pastores evengélicos por haberse capacitado en "el manejo del COVID19".

A la luz de lo anterior, entonces, queda claro que la religión, en la época de la COVID 19 , y como ya ha ocurrido en pasado, tiende a adaptarse al ambiente externo para sobrevivir, con todas las consecuencias que eso implica. Los escenarios futuros son, obviamente, impredecibles. La aspiración del presente número de Comparative Cultural Studies - European and Latin American Perspectives es, más bien, la de empezar a describir lo que está pasando ahora, a través de la mirada de algunos importantes estudiosos del fenómeno religioso contemporáneo, pertenecientes a diversas instituciones y desde diferentes puntos de vista disciplinarios.

\section{Presentación de los textos}

El primer artículo de Arnaldo Nesti, titulado "La storia di un’epidemia: dall'immaginario sfuggente, all'incertezza del domani” propone una reflexión profunda entorno a algunas de las consecuencias negativas de esta pandemia, como, por ejemplo, el problema 
de los "duelos suspendidos", y, al mismo tiempo, plantea la posibilidad/esperanza de que esta situación tan difícil pueda también estimular, en un cierto momento, sentimientos de solidaridad y amistad entre los seres humanos.

El texto de Enzo Pace se enfoca en las consecuencias negativas que ha implicado la pandemia para las Mega-iglesias, cuyos creyentes han tenido que renunciar a los eventos masivos presenciales en grandes auditorios, para adaptarse "a un servicio online, a escala nacional, para muchos fieles anónimos y lejanos”. Por lo tanto, según Pace, la pandemia implica, para estas iglesias, una doble problemática: por un lado, debilita la idea de un Dios que todo lo puede y, por otro, desmaterializa la presencia del enemigo, quien se vuelve invisible e intrusivo tanto que ya ningún líder carismático es capaz de contrarrestarlo.

Enzo Bova, en su artículo titulado "Tra saeculum e mysterium fidei. La chiesa cattolica in regime di lockdown", reflexiona sobre algunas de las principales consecuencias que está teniendo la curentena en la iglesia católica y, más específicamente, concentra sobre todo la atención en: la relación Estado-Iglesia, las estrategias de adaptación de los fieles y la institución religiosa, el papel del Papa Francisco, las preguntas que se le plantean a la institución y los posibles desenlaces en la futura forma de creer.

El texto de Giovanna Campani, partiendo de un hecho de crónica, concentra la atención en la relación entre ciencia y religión durante la pandemia, llegando a la conclusión de que, en este periodo, la única preocupación de los gobiernos occidentales ha sido cuidar la "vida biológica" de sus ciudadanos a través de medidas exclusivamente técnicas, "ignorando el bios, que concierne a las facultades superiores del hombre con sus necesidades espirituales".

Siguen dos textos que se enfocan en el contexto mexicano.

En el primero, Felipe Gaytán Alcalá y Ricardo Bernal sostienen que, como consecuencia de la pandemia, está surgiendo lo que ellos llaman Hogar-Mundo, es decir una dimensión existencial en el cual se mezclan "la esfera pública, privada e íntima en el interior de la casa”. El Hogar-Mundo, según los autores, tiende a favorecer el desarrollo de grupos religiosos definidos como Nuevos Puritanismos, los cuales pertenecen al campo del neoconservadurismo y, al mismo tiempo, se distancian de ello en el ámbito privado.

Jorge Valtierra Zamudio y Lorena Córdova-Hernández, en cambio, nos describen las formas de adaptación de la experiencia religiosa en algunas comunidades indígenas de México, en el estado de Chiapas y en el estado de Oaxaca. Los autores concluyen destacando que, a pesar de los problemas provocados por la pandemia, en estas comunidades la religión sigue jugando un papel fundamental.

El artículo de Diego Mauro y Mariano Fabris se enfoca en el contexto argentino, con el objetivo principal de entender cuál es el papel otorgado a la religión por los especialistas religiosos católicos y evangélicos, en la particular situación existencial consecuencia de la pandemia. Según Mauro y Fabris, el discurso de estos actores religiosos ha sido, en la mayoría de los casos, bastante secularizado, concentrando la atención en cuestiones mundanas tales como "la sociedad, la salud y la vida política, desprovista, además, de tintes apocalípticos y conspiracionistas".

Sigue el texto de Ciarocchi, en el cual el autor concentra la atención en los procesos de adaptación a la pandemia por parte de los devotos de la Virgen de la Limosna, patrona del pueblo de Biancavilla en Sicilia, quienes, durante la cuarentena, lograron mantener viva su devoción, sus relaciones personales y comunitarias, a través de las herramientas tecnológicas. 
El artículo de Daniela Turco nos propone un primer acercamiento a la fraternidad de "Amigos de Jesús Buen Pastor" (AGBP), "una nueva realidad eclesial" que se expresa en línea y que, durante el periodo de la cuarentena, ha visto crecer de manera muy significativa el número de sus seguidores. Las dos principales preguntas a las cuales intenta contestar la autora en su texto son: ¿qué buscan los creyentes/usuarios en esta nueva opción religiosa digital? ¿podrá, la fraternidad de "Amigos de Jesús Buen Pastor” convertirse en una nueva forma de pertenencia religiosa "en un contexto en el cual las religiones históricas parecen poco atractivas en comparación con las muchas alternativas de espiritualidad presentes en el mercado"?

En el último artículo, Davide Nicola Carnevale y Simona Fabiola Girneata analizan las prácticas y medidas adoptadas por las comunidades cristianas ortodoxas de Europa del Este en diáspora, para enfrentarse a la pandemia provocada por la COVID19. Más específicamente, utilizando las herramientas de la observación participante multi-situada, los dos autores nos proponen una comparación entre dos comunidades presentes en el contexto europeo: una en Francia y la otra en Italia.

Finalmente, en la sección Short notes, Giovanna Campani nos ofrece una breve descripción de algunos momentos de vida cotidiana en el estado brasileño de Ceara y en Río de Janeiro, con el objetivo de reflexionar críticamente sobre el distanciamiento social, una de las prácticas principales utilizadas en todo el mundo para enfrentarse a la pandemia.

¡Buena lectura!

\section{Referencias}

Bauman Z. (2007. Miedo líquido. La sociedad contemporánea y sus temores. Barcelona: Paidós Ibérica.

Beck, U. (1998). La sociedad del riesgo: hacia una nueva modernidad. Barcelona: Paidós.

Berger, P. (1977). El dosel sagrado. Elementos para una sociología de la religión. Buenos Aires: Amorrortu editores.

Berzano, L. (2014). Generatività della religione popolare. En Berzano, L. Castegnaro, A. Pace, E. (coord.), Religiosità popolare nella società post-secolare. Nuovi approcci teorici e nuovi campi di ricerca, Padova: Edizioni Messaggero.

De Martino, E. (2003). Sud e magia. Milano: Feltrinelli.

Giddens, A. (2014). Sociología, Madrid: Alianza Editorial.

Hervieu Léger, D. (2003). Il pellegrino e il convertito. La religione in movimento. Bologna: il Mulino.

Parsons, T. (1981). Il sistema sociale. Milano: Edizioni di Comunità.

Pastores reciben reconocimientos tras capacitación por COVID-19 (2020, 18 de diciembre). Vanguardia de Veracruz. Recuperado de: https://www.vanguardiaveracruz.mx/ pastores-reciben-reconocimientos-tras-capacitacion-por-covid-19/

Velasco, M. (2021, 17 de febrero). Con ceniza en bolsa y misas grabadas, así vive Edomex el Miércoles de Ceniza. Excelsior. Recuperado de: https://www.excelsior.com.mx/ comunidad/con-ceniza-en-bolsa-y-misas-grabadas-asi-vive-edomex-el-miercolesde-ceniza/ 1433351

Voyé, L. (1999). Secularization in a Context of Advanced Modernity. Sociology of Religion, (60) 3, 275-288. 